\title{
RHO-OMEGA INTERFERENCE IN $\overline{\mathrm{p}} \mathrm{p} \rightarrow 4 \pi$ AT $1.6-2.2 \mathrm{GeV} / c^{*}$
}

J. W. CHAPMAN, J. DAVIDSON, R. GREEN, J. LYS, B. ROE and J. C. VANDER VELDE

The Universily of Michigan, Ann Arbor, Michigan 4810.4

Received 23 July 1970

(Revised manuscript received 14 September 1970)

\begin{abstract}
The $\pi^{+} \pi^{-}$mass spectrum from over 3000 events of the reaction pp $\rightarrow \pi^{+} \pi^{+} \pi^{-} \pi^{-}$at $1.63-2.20 \mathrm{GeV} / c$ shows evidence for $\rho-\omega$ interference. If we allow for $\rho$-background interference the evidence is weak in our total data, but is strong when we make a particular cut on the momentum transfer, from the proton or antiproton to the $\pi^{+} \pi^{-}$. A fit to the data with this cut gives a lower limit on the branching ratio $\omega \rightarrow \pi^{+} \pi^{-}$to $\omega \rightarrow \pi^{+} \pi^{-} \pi^{\circ}$ of $1.9 \%(95 \%$ confidence level).
\end{abstract}

\section{INTRODUCTION}

The $\omega-\rho$ interference effect in $\pi^{+} \pi^{-}$mass spectra has been observed in $\pi \mathrm{p}[1,2], \mathrm{K}^{-} \mathrm{p}[3]$, and $\bar{p} \mathrm{p}[4]$ experiments in bubble chambers, in an $\mathrm{e}^{+} \mathrm{e}^{-}$ colliding beam experiment [5] and in photoproduction from carbon [6]. In the $\bar{p}$ p case, Allison et al. saw an effect in 1448 events of the channel $\overline{\mathrm{p}}-\pi^{+} \pi^{+} \pi^{-} \pi^{-}$at incident $\overline{\mathrm{p}}$ momentum $1.26-1.65 \mathrm{GeV} / \mathrm{c}$. We have looked for this effect in 3135 events of the same channel at the slightly higher incident $\overline{\mathrm{p}}$ momentum interval $1.63-2.20 \mathrm{GeV} / c$.

\section{EXPERIMENTAL DETAILS}

From an antiproton exposure in the Argonne-MURA 30-inch hydrogen bubble chamber covering six incident momenta from 1.63 to $2.20 \mathrm{GeV} c$, we have measured 50000 four-prong events to obtain our four-pion sample. The $\pi^{+} \pi^{-}$mass spectrum from these $4 \pi$ events is shown in fig. 1 . The average $4 \pi$ cross section in this momentum interval is $1.8 \pm 0.1 \mathrm{mb}$, and a prelim. inary fit of the $\pi^{+} \pi^{-}$mass spectrum to simple incoherent Breit-Wigners and phase space indicates the following approximate percentage contributions of various channels - $10 \% \rho \rho, 40 \% \rho f, 20 \% \rho \pi \pi, 20 \% \mathrm{f} \pi \pi, 10 \% 4 \pi$. Events that fit $\overline{\mathrm{p}} \mathrm{p} \rightarrow \pi^{+} \pi^{+} \pi^{-} \pi^{-} \pi^{\mathrm{O}}$ show copious $\omega$-production: we estimate we have 2147 $\omega \pi \pi$ events (including $\omega \rho, \omega f$ ), corresponding to $\approx 1.2 \mathrm{mb}$.

* Work supported by the Lis Atomic Energy Commission. 


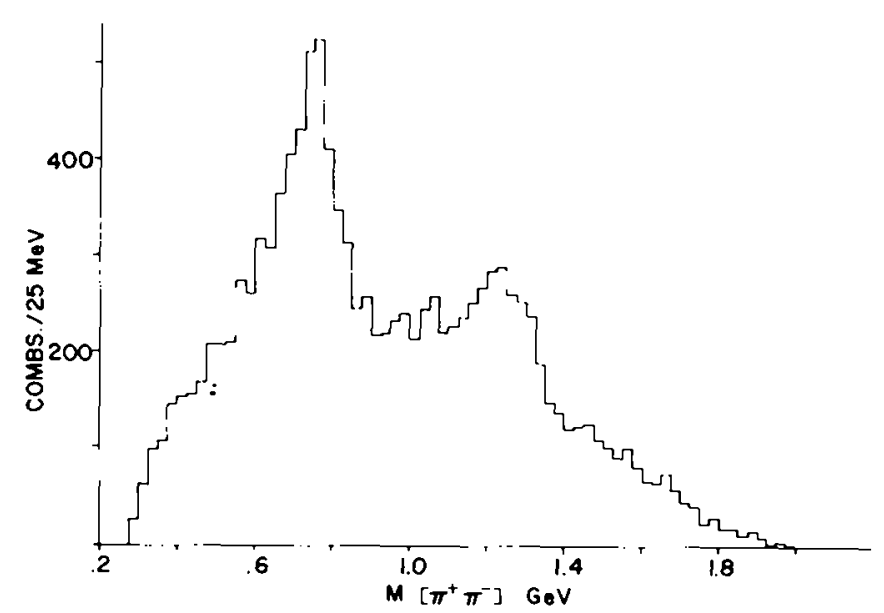

Fig. 1. The $\pi^{+} \pi^{-}$mass spectrum for all $\pi^{+} \pi^{+} \pi^{-} \pi^{-}$events (4 combinations per event).

A study of our errors, and the width of the $\omega$-peak in the $\pi^{+} \pi^{-} \pi^{\mathrm{O}}$ mass plot, shows that our $\pi^{+} \pi^{-}$mass resolution near $780 \mathrm{MeV}$ is $11 \pm 2 \mathrm{MeV}$ FWHM. Therefore, in the fits to our $\pi^{+} \pi^{-}$mass spectra to be described below, we always fold in a Gaussian error function with $\sigma=5 \mathrm{MeV}$. We also assume in our fits a mass and width for the $\omega$ of $783.7 \mathrm{MeV}$ and 12.7 MeV, respectively [7]. We have checked that our fits are insensitive to small changes in the values of $\sigma$ and the $\omega$-mass. Thus in fitting our total data to eq. (8) below, $x^{2}$ changes by 0.5 if we take $\sigma$ anywhere from 4 to $7 \mathrm{MeV}$ and $m_{\omega}$ from 782.7 to $784.7 \mathrm{MeV}$.

To investigate possible $\omega-\rho$ interference in our data, we have fitted our $\pi^{+} \pi^{-}$mass spectrum, from $570 \mathrm{MeV}$ to $950 \mathrm{MeV}$ in $10 \mathrm{MeV}$ bins, to various theoretical expressions, with and without various cuts on our data.

\section{FITS TO SIMPLE RHO-OMEGA INT IRFERENCE}

Allison et al. [4] fit their pp data to the expression

$$
\begin{aligned}
& \frac{\mathrm{d} N}{\mathrm{~d}} \bar{m}=\operatorname{PS}\left[A_{p s}+A_{\rho}^{2}\left|\mathrm{BW}_{\rho}\right|^{2}+\left.A_{\omega^{\mid}}^{2} \mathrm{BW}\right|_{\omega} ^{2}+2 \alpha A_{\omega} A_{\rho} \operatorname{Re}\left(\mathrm{e}^{i \varphi} \mathrm{BW}_{\omega} \mathrm{BW}_{\rho}^{*}\right)\right] \text {, } \\
& \mathrm{BW}_{\rho}=-\frac{\Gamma^{1 / 2}}{\left(m_{\rho}^{2}-m^{2}\right)-i m_{\rho} \Gamma}, \\
& \Gamma=\Gamma_{\rho}{ }^{-}{ }^{m}{ }^{-}\left(\frac{q}{q_{\rho}}\right)^{3} \\
& \mathrm{BW}_{\omega}=\frac{\Gamma_{\omega}^{1 / 2}}{\left(m_{\omega}^{2}-m^{2}\right)-i m_{\omega} \Gamma_{\omega}} .
\end{aligned}
$$


Here, PS is phase space (2 pions out of 4 pions), $\alpha$ is a coherence factor, $\alpha \leqslant 1.0$, and $q$ is the momentum of either pion in the $\pi^{+} \pi^{-}$c.m.. Re stands for real part of.

If only one process contributes to $\omega$ - and $\rho$-production, then $\varphi$ is the relative phase of the amplitudes to produce $\pi^{+} \pi^{-}$via $\rho$ - and $\omega$-production amplitudes, and $\alpha=1$. In general, eq. (1) can be rewritten as a sum over many processes:

$$
\frac{\mathrm{d} N}{\mathrm{~d} m}=\operatorname{PS}\left[A_{\mathrm{p} . \mathrm{s} .}+\sum_{i}\left|A_{\rho i} \mathrm{BW}_{\rho}+\mathrm{e}^{i \varphi_{i}} A_{\omega i} \mathrm{BW}_{\omega}\right|^{2}\right]
$$

and then:

$$
\begin{gathered}
\sum_{\tan \varphi} A_{\rho i} A_{\omega i} \sin \varphi_{i} \\
\sum_{i}^{i} A_{\rho i} A_{\omega i} \cos \varphi_{i}
\end{gathered}
$$

We have fitted our data to eq. (1), taking as free parameters $m_{\rho}, \Gamma_{\rho}$, $A_{\omega}, \varphi, \alpha, A_{\rho}$. Here $A_{\mathrm{p} . \mathrm{s}}$. is fixed by the overall normalization. If we set $A_{\omega}$ to zero, we get $\chi^{2}=65$ (34 degrees of freedom), with $m_{\rho}=746 \mathrm{MeV}$, $\Gamma \rho=132 \mathrm{MeV}$. With non-zero $A \omega$, we get $\chi^{2}=49$, with $m_{\rho}=749 \mathrm{MeV}$, $\Gamma_{\rho}=179 \mathrm{MeV}, \varphi=100^{\circ}, \alpha=0.9$, and an $\omega$ branching ratio $R=2.7 \%$. Here $R$ is the ratio of $\omega \rightarrow \pi^{+} \pi^{-}$decays to $\omega \rightarrow \pi^{+} \pi^{-} \pi^{0}$ decays, with

$$
N\left(\omega \rightarrow \pi^{+} \pi^{-}\right)=\int \operatorname{PS} A_{\omega}^{2}\left|\mathrm{BW}_{\omega}\right|^{2} \mathrm{~d} m \text {. }
$$

Thus introducing the three $\rho-\omega$ interference parameters $A_{\omega}, \alpha, \varphi$ (although only two are independent [4]), reduced $x^{2}$ by 16 . Our $\rho$-width of $179 \mathrm{MeV}$ is rather large (Allison et al. also found a large width, $\Gamma_{\rho} \approx 180$ $\mathrm{MeV}$ ). If we restrict $\Gamma_{\rho}$ to less than $145 \mathrm{MeV}$, we get $x^{2}=51$, with the other parameters essentially unchanged.

If we require $\Delta x^{2}-2.7$, we find $\alpha=0.4$ and $R$ in the range $0.75 \%$ to $40 \%$. Allison et al. found $\alpha=0.4$ also, and $R=1.4 \%$ to $42 \%$, for $\Delta x^{2}=2.7$. In addition, they found $\varphi=60^{\circ}-90^{\circ}$ if [5] $R=1.3 \%$ to $6.0 \%$, while our corresponding range is $95^{\circ}-125^{\circ}$.

Thus the results of fitting our total data to this simple $\rho-\omega$ interference model are very similar to the results of Allison et al. .

\section{FITS WITH RHO-BACKGROUND INTERFERENCE}

Another expression with which to fit the $\pi^{+} \pi^{-}$mass spectrum has been given by Flatte [3] and used by Abramovitch et al. [2]

$$
\begin{aligned}
\frac{\mathrm{d} N}{\mathrm{~d} m}= & \left\{\alpha_{1}+\alpha_{2}|\mathrm{BW} \rho|^{2}+\alpha_{3}\left|\mathrm{BW}_{\omega}\right|^{2}+\alpha_{4} \operatorname{Re} \mathrm{BW}_{\rho}+\alpha_{5} \operatorname{Re} \mathrm{BW}_{\omega}\right\} \\
& \times\left\{1+\alpha_{6}\left(m-m_{\omega}\right)+\alpha_{7}\left(m-m_{\omega}\right)^{2}\right\} .
\end{aligned}
$$

This expression allows for coherence between the $\rho$-and $\omega$-amplitudes and the non-resonant background. The approximations are made that: 


$$
\begin{aligned}
& \mathrm{BW}_{\rho} \mathrm{BW}_{\omega}=\text { const. } \mathrm{BW}_{\omega}, \\
& \operatorname{Im}(\mathrm{BW})=\text { const. }|\mathrm{BW}|^{2} .
\end{aligned}
$$

Eq. (8) becomes the same as eq. (1) if we set $\alpha_{4}$ to zero ( and make the approximations (9) and (10)). In our case, we certainly have some background under the $\rho$-peak, and have no reason to suppose it contains no $l=1$ component which could interfere. Hence we refitted our data using eq. (8). In addition, we used the Breit-Wigner amplitudes of ref. [2], which involves replacing the numerators of eqs. (2) and (4) with $m_{\mathrm{X}} \Gamma_{\mathrm{X}} q / q_{\mathrm{X}}, \mathrm{x}=\rho$ or $\omega$.

Preliminary fits produced values of the "background" parameters $\alpha_{6}$ and $\alpha 7$ close to those required to fit two-body out of four-body phase space. Hence on our later fits we fixed $\alpha 6$ and $\alpha 7$ to these phase-space values. In addition, we have constrained the $\rho$ mass and width to lie within the probable limits of the Particle Properties Tables [7]: $m_{\rho}=755-775 \mathrm{MeV}$, $\Gamma_{\rho}=105-145 \mathrm{MeV}$. A fit to our total data with eq. (8), without limits on $m_{\rho}$ and $\Gamma_{\rho}$, gave $773 \mathrm{MeV}$ and $145 \mathrm{MeV}$, respectively.

We have fitted eq. (8) to numerous subsets of our data obtained by making cuts on the incident $\ddot{\mathrm{p}}$ momentum $P$, the momentum transfer $t$, and the recoil mass $M$. We define $t$ as the squared four-momentum transfer between the $\pi^{+} \pi^{-}$system and either the $\mathrm{p}$ or the $\overline{\mathrm{p}}$, taking the algebraic la rger of the alternatives. For each subset, we made four fits: (a) with $\alpha_{3}$, $\alpha_{4}, \alpha_{5}$, set to zero - i.e., incoherent $\rho$ plus background; (b) $\alpha_{3}, \alpha_{5}$, set to zero - $\rho$-plus-background with interference between them; (c) $\alpha 4$ set to zero - incoherent background, as in cq. (1): (d) $\alpha_{2}, \alpha_{3}, \alpha_{4}, \alpha_{5}$, all nonzero. The $x^{2}$ values for the fits to some of our data sets are given in table 1. The fits are normalized to the number of mass combinations in each data set (this fixes the parameter $\alpha_{1}$ ). There is no restriction on the number of mass combinations any one event can contribute to a particular data set ${ }^{*}$.

The fit to the total data gives evidence that the re is in fact interference between the $\rho$ and the background whether or not we admit $\omega$ - $\rho$ interference $x^{2}$ decreases by 27 from fits (a) to (b), or by 10 from (c) to (d), with the introduction of one new parameter ${ }^{* *}$. If we admit $\rho$-background interference, then the evidence for $\omega-\rho$ interference in the total data is not so good $-x^{2}$ decreases by 7 with two new parameters. Nearly all of this decrease arises from the $790-800 \mathrm{MeV}$ bin. Fits to the total data are shown in fig. $2 a$.

* In all our fits we take the error on the number of mass combinations in any bin to be the square root of the number of combinations. This is an approximation in that some events contribute more than one combination to the mass spectrum we fit. 'To do anything else is difficult, and we believe the effect on our fits is small.

** Of course this decrease in $x^{2}$ could be due to some different mechanis $m$ which happens to have a similar effect on the $\pi^{+} \pi^{-}$mass spectrum. For instance, if we write the matrix element for the reaction $\mathrm{pp} \rightarrow \rho \pi^{+} \pi^{-}$as a sum of four BreitWigner amplitudes (so as to be symmetric in the interchange of like pions), the resulting $\pi^{+} \pi^{-}$mass spectrum can be approximated by the expression $\mathrm{d} N / \mathrm{d} m=\operatorname{PS}\left[a_{1}: a_{2}\left|3 W_{\rho}\right|^{2}+a_{3} \mathrm{Re}(\mathrm{BW} \rho)\right]$ where $a_{3}$ is non negligible (but has opposite sign to that required to fit our total data). 
Table 1

The $x^{2}$ values from least-squares fits of eq. (s) to various selections of our data.

\begin{tabular}{|c|c|c|c|c|c|}
\hline Data selection & $\begin{array}{l}\text { Fit } \\
\text { (a) }\end{array}$ & $\begin{array}{r}\text { Fit } \\
\text { (b) }\end{array}$ & $\begin{array}{l}\text { Fit } \\
\text { (c) }\end{array}$ & $\begin{array}{l}\text { Fit } \\
\text { (d) }\end{array}$ & $N$ \\
\hline All data & 69 & 42 & 46 & 36 & 5200 \\
\hline$l^{P}-1.95,2.20$ & 43 & 42 & 40 & 40 & 1328 \\
\hline$P^{P}=1.83,1.89$ & $3 \overline{5}$ & 25 & 29 & 23 & 1869 \\
\hline$P^{\prime}=1.63,1.77$ & 45 & 28 & 29 & 23 & 2003 \\
\hline$-t=0.0-0.3$ & 75 & 56 & 51 & 47 & 2030 \\
\hline$-t=0.3-0.6$ & 43 & 43 & 40 & 40 & 1409 \\
\hline$-t=0.6-0.9$ & 33 & 32 & 32 & 32 & 898 \\
\hline$-t=0.9-1.2$ & 3.5 & 35 & 35 & 34 & 469 \\
\hline$-t=0.0-0.1$ & 45 & 42 & 42 & 39 & 651 \\
\hline$-t-0.1-0.2$ & 57 & $4 \overline{5}$ & 50 & 45 & $74 i$ \\
\hline$-t=0.2-0.3$ & 62 & 52 & $3 \mathrm{l}$ & 31 & 633 \\
\hline $\left.\begin{array}{l}P=1.9 \overline{5}, 2.20 \\
-t=0.0-0.3\end{array}\right\}$ & 51 & 44 & 47 & 44 & 458 \\
\hline $\left.\begin{array}{l}P=1.83,1.89 \\
-t=0.0-0.3\end{array}\right\}$ & 63 & 52 & 50 & 45 & 703 \\
\hline $\left.\begin{array}{l}P=1.63,1.77 \\
-t=0.0-0.3\end{array}\right\}$ & 43 & 36 & 33 & 32 & 869 \\
\hline$M=0.67-0.81$ & 68 & 67 & 67 & 67 & 753 \\
\hline$M=1.135-1.325$ & 37 & 36 & 27 & 26 & 1287 \\
\hline $\left.\begin{array}{l}M=0.81-1.135 \\
M<0.67, M>1.325\end{array}\right\}$ & 75 & 32 & 54 & 29 & 3160 \\
\hline $\left.\begin{array}{l}P-1.63-1.89 \\
-t=0.0-0.3\end{array}\right\}$ & 69 & 54 & 45 & 42 & 1572 \\
\hline $\begin{array}{l}\left.\begin{array}{l}M=1.135-1.325 \\
-t=0.0-0.3\end{array}\right\}\end{array}$ & 47 & 46 & 40 & 36 & ¡37 \\
\hline
\end{tabular}

Fits (a), (b), (c), (d), correspond to (a) $\rho$ plus incoherent background, (b) coherent $\rho$ and background, (c) coherent $\rho$ and $\omega$ plus incoherent background, (d) coherent $\rho$ and $\omega$ and background (sec text; "coherent" means any degree of coherence - including complete incoherence). Here $P$ is the incident momentum in GeV $/ c ; t$ is the momentum transfer in $(\mathrm{GeV} / c)^{2}$ - see text; $M$ is the recoil mass in GeV and $N$ is the number of $\pi^{+\cdot} \pi^{-}$mass combinations involved.

Our strongest evidence for $\rho-\omega$ interference comes from the $t=-0.2$ to $-0.3(\mathrm{GeV} / \mathrm{c})^{2}$ data, in which $x^{2}$ decreases by 20 between fits (b) and (d). Clearly, when we make $t$-cuts we allow interference between different angular momenta, which must disappear when integrated over all angles. The $t=-0.2$ to -0.3 data and fits are shown in fig. $2 \mathrm{~b}$.

From our fits to the total data and to the $t=-0.2$ to -0.3 data, we have calculated lower limits for the branching ratio $R$ of $\omega \rightarrow \pi^{+} \pi^{-}$to $\omega \rightarrow \pi^{+} \pi^{-} \pi^{\circ}$. 

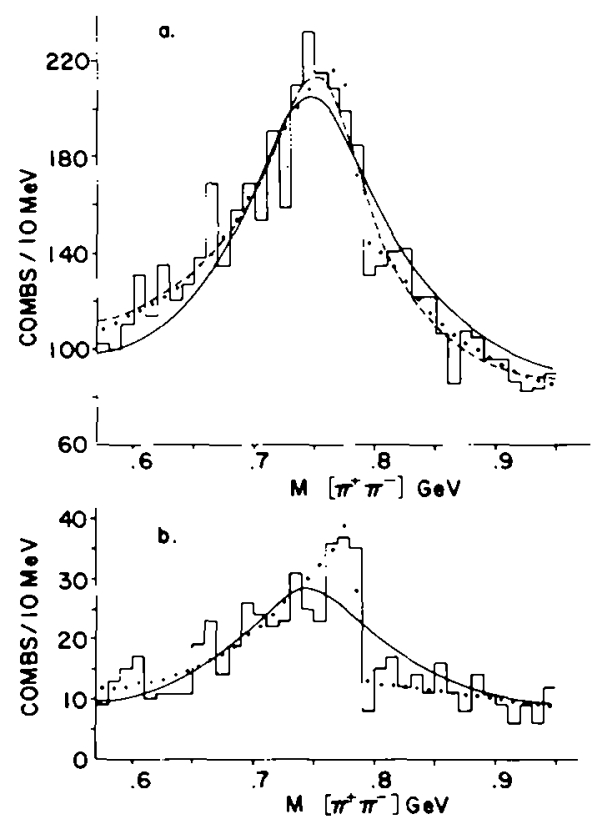

Fig. 2. (a) The part of the $\pi^{+} \pi^{-}$mass spectrum used in our fits. The solid curve is fit (a) to eq. (8), the dashed curve is fit (b). the solid circles are for fit (d) (see text). (b) The subset of (a) with squared four-momentum transfer to the $\pi^{+} \pi^{-}$system in the range -0.2 to $-0.3(\mathrm{GeV} / \mathrm{c})^{2}$. The solid curve and solid circles are fits (a) and (d). respectively, to eq. (8).

The fitted values of $\alpha_{1-5}$ can in general accomodate a range of $\omega$ branching ratios; the lower limit comes from assuming complete coherence of the $\rho$, $\omega$ and background amplitudes, and is obtained from [2,3]

$$
\begin{gathered}
A=b-\left(b^{2}-\alpha_{3}^{2}-\alpha_{5}^{2}\right)^{1 / 2}, \\
b=2 \alpha_{1}+2 \alpha_{2}\left|\mathrm{BW}_{\rho}\right|_{m=m_{\omega}}^{2}+\alpha_{3}+2 \alpha_{4}\left[\operatorname{Re~BW}_{\rho}\right]_{m=m_{\omega}}, \\
N\left(\omega \rightarrow \pi^{+} \pi^{-}\right)=A \int \mid \mathrm{BW}_{\omega \mid}{ }^{2} \mathrm{~d} m .
\end{gathered}
$$

The results are $R=0.5 \%$ and $5.3 \%$, respectively. By making fits with various fixed values of the quantity $A$, as defined in eqs. (11), (12), we can get a plot of $x^{2}$ versus $A$ or $R$, and hence a $95 \%$ confidence level lower limit on the value of $R$ (corresponding to $\Delta \chi^{2}=4.0$ ). The results are $0.04 \%$ and $1.9 \%$, again respectively for the total data and for the $t=-0.2$ to -0.3 data.

Inspection of table 1 shows that when we make various $t$-cuts on our data, we find a significant $\rho-\omega$ interference signal, as measured by the decrease in $x^{2}$ between fits (b) and (d), only for the $t=-0.2$ to -0.3 range. This variation with $t$ can be accomodated simply by writing the relative phase of the $\rho$ and $\omega$ amplitudes for each contributing process as an ap- 
propriate function of $t$. The relatively high value of $R$ for the $t=-0.2$ to -0.3 data, compared to the results of Augustin et al. [5] and Biggs et al. [6] (the latter gives a branching ratio of $0.80 \pm 0.25 \%$ ), implies one dominant process, or equivalently that all the different (incoherent) processes have similar magnitude and phase for the $\rho$-and $\omega$-amplitude ratios. To study further this $t$-dependence, we have computed the density matrix elements as a function of $t$ for the $\rho$ - and $\omega$-decays (using the $\pi^{+} \pi^{-} \pi^{0}$ decay mode of the $\omega)$. Within our statistics the matrix elements are similar for the two particles, and show no striking $t$-dependence. The two cross sections versus $t$ are also similar, each showing a peak at $t \approx-0.25$, with a slow fall off at $t=-0.2$ and a faster fall off at $t=-0.3$ (our allowed $t$ range for a $\rho$ is $\approx+0.05$ to $\left.-1.50(\mathrm{GeV} / \mathrm{c})^{2}\right)$.

We find no evidence for a $\rho-\omega$ interference signal at our highest pair of momenta, nor when the recoil mass is not in the $f$-meson region. Hence our data are consistent with a $\rho-\omega$ interference effect in the $\rho$ f channel at incident $\overline{\mathrm{p}}$ momenta $1.6-1.9 \mathrm{GeV} / c$. Our limited statistics prevent us from making any stronger statement. We note that the $\omega$ f cross section does not show any strong variation as a function of $\bar{p}$ momentum.

In making cuts on our data, we could use $|\cos \theta|$ in place of $t$, where $\theta$ is the c.m. production angle of the $\pi^{+} \pi^{-}$system with respect to the proton or antiproton. These two variables a re equivalent only for fixed recoil mass. We have looked at our data for various $|\cos \theta|$ intervals, and find a significant change in $x^{2}$ between fits (b) and (d) to the data only for the interval $\mid \cos \theta=0.6$ to 0.8 (when $x^{2}$ are 61 and 45 , respectively). This is to be expected from our previous fitting, since for the $\rho$ f channel $t=-0.25$ corresponds to $|\cos \theta| \approx 0.7$.

We have also fitted the data of Allison et al. [4], as deduced from their fig. 1 , to eq. (8), for $m\left(\pi^{+} \pi^{-}\right)=570-950 \mathrm{MeV}$, using the same phase space and $m_{\rho}, \Gamma \rho$, bounds as for our data. Fits (a) - (d) give $\chi^{2}$ values of 51,38 , 36,32 respectively. Fit (d) gives $R=0.66 \%$. This suggests that in fact the evidence for $\rho-\omega$ interference in their total data is weak - as it is in ours once we allow $\rho$-background interference.

\section{OTHER INTERFERING AMPLITUDES}

We have investigated the possibility that some other simple interference can produce the same effect on the $\pi^{+} \pi^{-}$mass spectrum as $\rho-\omega$ interference.

The possible association of our effect with the $\rho$ channel suggests we look at interfering $\rho$ - and f-amplitudes. We have generated $4 \pi$ events weighted with the square of matrix elements of the form

$$
\begin{aligned}
M & =\mathrm{BW}_{\rho 13}+\mathrm{BW}_{\rho 14}+\mathrm{BW}_{\rho 23}+\mathrm{BW}_{\rho 24}+c \mathrm{e}^{\mathrm{i} \gamma}\left(\mathrm{BW}_{\rho 13} \mathrm{BW}_{\mathrm{f} 24}\right. \\
& \left.+\mathrm{BW}_{\rho 14} \mathrm{BW}_{\mathrm{f} 23}+\mathrm{BW}_{\rho 23} \mathrm{BW}_{\mathrm{f} 14}+\mathrm{BW}_{\rho 24} \mathrm{BW}_{\mathrm{f} 13}\right),
\end{aligned}
$$

where pions 1 and 2 have the same charge, $B W_{\rho}$ is the same Breit-Wigner amplitude as used in eq. (8), $\mathrm{BW}_{\mathrm{f}}$ is a Breit-Wigner amplitude for the 
$\mathrm{f}$-meson, and is exactly similar to $\mathrm{BW} \rho$ apart from a fifth-power momentum dependence for the width (cf. eq. (3)) and $c$ and $\gamma$ a re variable parameters.

None of the resulting $\pi^{+} \pi^{-}$mass distributions (we varied $c$ and $\gamma$ ) reproduced the sharp drop near $790 \mathrm{MeV}$ seen in our data and in our $\rho-\omega$ interference fits.

We have also tried matrix elements with interfering $\rho \rho$ and $\rho \pi \pi$ amplitudes, written exactly analagously to eq. (14) above. Again we could not produce a sharp drop near $790 \mathrm{MeV}$.

\section{CONCLUSION}

We conclude that in our pp $\rightarrow \pi^{+} \pi^{+} \pi^{-} \pi^{-}$data we see evidence of $\rho-\omega$ interference. The interference is a function of the momentum transfer $t$, having a maximum signal at $-t=0.2-0.3 .(\mathrm{GeV} / c)^{2}$, and is consistent with being all in the of channel. We find that fits to the data may be considerably improved by inclusion of $\rho$-background interference. Our best value for the branching ratio $\omega \rightarrow \pi^{+} \pi^{-}$to $\omega \rightarrow \pi^{+} \pi^{-} \pi^{0}$ is $-1.9 \%$ (95\% CL).

A check that we are really observing $\rho-\omega$ interference could come from looking at the reaction $\overline{\mathrm{p}} \mathrm{n} \rightarrow \pi^{+} \pi^{-} \pi^{-} \pi^{\circ}$, where the interference signal in the $\pi^{+} \pi^{-}$mass spectrum should be suppressed in the total data, but not necessarily in $t$-selected data (note also this reaction contains no $\rho$ of channel). This follows because here the $\omega \pi^{-} \pi^{0}$ and $\rho \pi^{-} \pi^{0}$ systems have the same $I$ value $(I=1)$ but different $G$-parities, and hence for the same $\bar{p}$ spin state, different parities. The strong $t$-dependence of our effect and the high degree of coherence implied by the fit at $-t=0.2$ to 0.3 are puzzling aspects of our data that may induce skepticism about our $\rho-\omega$ interference interpretation.

We are indebted to Dr. F. Schweingruber, Dr. L. Voyvodic, and the crew of the 30-inch bubble chamber at Argonne National Laboratory. We also thank Professor C. T. Murphy, who was involved in earlier aspects of this experiment.

\section{REFERENCES}

[1] G. Goldhaber, W. R. Butler, D. G. Coyne, B. H. Hall, J. N. MacNaughton and G.H. Trilling, Phys. Rev. Letters 23 (1969) 1351.

[2] M. Abramovitch, H. Blumenfeld, F. Bruyant, V.Chaloupka, S. L.Chung, J.Diaz and F. Martin, Nucl. Phys. B20 (1970) 209.

[3] S. M. Flatte, Phys. Rev. D1 (1970) 1.

[4] W. W. M. Allison, W. A. Cooper, T. Fields and D.S. Rhines, Phys. Rev. Letters $24(1970) 618$.

[5] J.E.Augustin, D. Benaksas, J.Buon, F. Fulda, V. Gracco, J.Haissinski, D. I.alande, F. Laplanche, J. Lef rancois, P. Lehmann, P.C. Marin, J.Perez-y-Jorba, F. Rumpf and E.Silva, Nuovo Cimento Letters 2 (1969) 214.

[6] P..J. Biggs, R.W. Clifft, E. Gabathuler, P. Kitching and R.E. Rand, Phys. Rev. Letters 24 (1970) 1201.

[7] Particle Data Group, Rev. Mod. Phys. 42 (1970) 87. 